\title{
Characterization of Materials for Energy Storage and Production by Helium Ion Microscopy Coupled to Secondary Ion Mass Spectrometry
}

\author{
Jean-Nicolas Audinot ${ }^{1}$, Jelena Lovric ${ }^{1}$, and Tom Wirtz ${ }^{1 *}$
}

1. Advanced Instrumentation for Ion Nano-Analytics, Department of Materials Research and Technology, Luxembourg Institute of Science and Technology (LIST), Belvaux, Luxembourg.

* Corresponding author: tom.wirtz@list.lu

Materials for producing and storing energy have become the focus of intensive research and their development requires a wide range of tools and methodologies for an accurate and in-depth physicochemical characterization. In particular, instruments and methodologies allowing a multi-modal characterization based on correlative approaches are of utmost importance.

In 2015, we first presented a Secondary Ion Mass Spectrometry (SIMS) system which we specifically developed for the Zeiss ORION NanoFab Helium Ion Microscope (HIM) [1]. This SIMS system is based on (i) specifically designed secondary ion extraction optics coupled with post-acceleration transfer optics, providing maximized extraction efficiency while keeping a finely focussed primary ion beam for highest lateral resolution, (ii) a compact floating double focusing magnetic sector mass spectrometer allowing operation in the DC mode at full transmission (and hence avoiding duty cycles like in TOF systems that either lead to very long acquisition times or, for a same acquisition time, intrinsically limit the sensitivity) and (iii) a specific detection system allowing the detection of several masses in parallel. We have demonstrated that our instrument is capable of producing (i) mass spectra with mass resolution $\mathrm{m} / \Delta \mathrm{m}=400-500$, (ii) very local depth profiles and (iii) elemental SIMS maps with lateral resolutions down to $12 \mathrm{~nm}$ [1-6]. Furthermore, HIM-SIMS opens the way for in-situ correlative imaging combining high resolution SE images with elemental and isotopic ratio maps from SIMS [2-4,7]. This approach allows SE images of exactly the same zone analysed with SIMS to be acquired easily and rapidly, followed by a fusion between the SE and SIMS data sets [7].

Here, we will review the instrument performance and present a number of examples taken from various fields of applications, with a special emphasis on battery materials and photovoltaic materials.

Nanoscale mapping of cathode materials of batteries was achieved by multi-modal correlative microscopy. Mapping nanoscale distributions of carbon, lithium, manganese and cobalt reveals the micro-structural changes as a consequence of electrochemical reactions. Additionally, it allows identifying Li "trapping" sites within the structure that control materials properties and open the way towards designing better Li-ion cathodes.

Correlative HIM-SIMS is also particularly well adapted for the nanoscale characterization of perovskite and CIGS solar cells. While secondary electron imaging allows the characterization of the morphology of the films, the size of the grains or the presence of defects, SIMS imaging provides the chemical information about the chemical homogeneity, the grain boundary composition and the chemical identification of nano-structures such as pinholes $[5,6,8]$. 


\section{References:}

[1] T Wirtz, P Philipp, J-N Audinot, D Dowsett, S Eswara, Nanotechnology 26 (2015), p. 434001.

[2] T Wirtz, D Dowsett and P Philipp in "Helium Ion Microscopy", ed. G Hlawacek and A Gölzhäuser (Springer, Switzerland), p. 297.

[3] D Dowsett, T Wirtz, Anal. Chem. 89 (2017), p. 8957.

[4] T Wirtz, O De Castro, J-N Audinot, and P Philipp, Annual Review of Analytical Chemistry 12 (2019).

[5] P Gratia et al., J. Am. Chem. Soc. 138 (2016), p. 15821.

[6] P Gratia et al., ACS Energy Lett. 2 (2017), p. 2686.

[7] F Vollnhals et al., Anal. Chem. 89 (2017), p. 10702.

[8] This work was co-funded by the National Research Fund Luxembourg (FNR) within the framework of the SOLAR4D project through grant INTER/SNF/16/11534230.
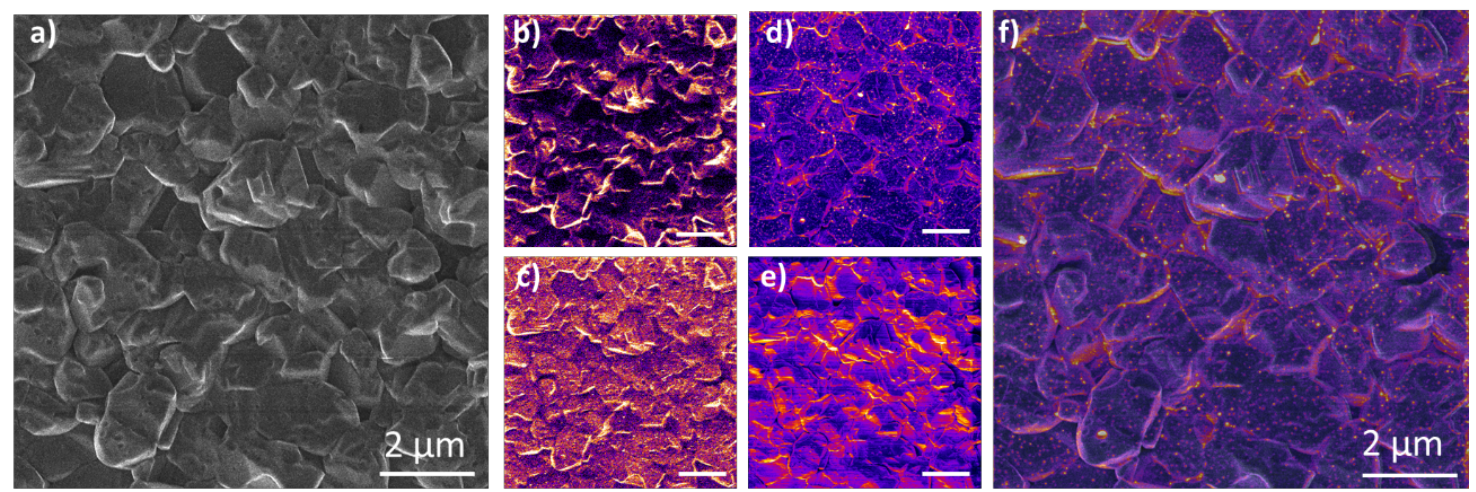

Figure 1. CIGS solar cell. a) Secondary electron image obtained on the Helium Ion Microscope showing the morphology and structure of the active layer. Secondary Ion Mass Spectrometry (SIMS) imaging on the HIM of oxygen (b), selenium (c), sodium (d) and indium (e). Overlay (f) of the sodium SIMS image and the secondary electron image. Scale bar: $2 \mu \mathrm{m}$. 